\title{
Germanica
}

\section{Max Reinhardt, Firmin Gémier, Jacques Copeau : influences et analogies}

Max Reinhardt, Firmin Gémier, Jacques Copeau: Einflüsse und Ähnlichkeiten

\section{Philippe Baron}

\section{(2) OpenEdition}

\section{Journals}

Édition électronique

URL : http://journals.openedition.org/germanica/582

DOI : 10.4000/germanica.582

ISSN : 2107-0784

\section{Éditeur}

Université de Lille

\section{Édition imprimée}

Date de publication : 1 décembre 2008

Pagination : 161-172

ISBN : 978-2-913857-22-6

ISSN : 0984-2632

\section{Référence électronique}

Philippe Baron, «Max Reinhardt, Firmin Gémier, Jacques Copeau : influences et analogies », Germanica [En ligne], 43 | 2008, mis en ligne le 01 décembre 2010, consulté le 06 octobre 2020. URL : http:// journals.openedition.org/germanica/582 ; DOI : https://doi.org/10.4000/germanica.582

Ce document a été généré automatiquement le 6 octobre 2020.

(ㄷ) Tous droits réservés 


\title{
Max Reinhardt, Firmin Gémier, Jacques Copeau : influences et analogies
}

Max Reinhardt, Firmin Gémier, Jacques Copeau: Einflüsse und Ähnlichkeiten

\author{
Philippe Baron
}

1 Dans cette contribution, je me propose d'étudier les similitudes qui existent entre certaines réalisations de Max Reinhardt d'un côté, de Firmin Gémier et de Jacques Copeau de l'autre. Je me poserai la question de savoir s'il s'agit d'analogies ou d'influences.

\section{Max Reinhardt}

2 Max Reinhardt ${ }^{1}$, né en 1873, entre en 1894 dans la troupe du Deutsches Theater dirigé par Otto Brahm. Otto Brahm, qui avait fondé en 1889 la Freie Bühne sur le modèle du Théâtre libre d'Antoine, se rattachait au courant naturaliste. Mais Max Reinhardt ressent cependant assez vite le besoin de s'en évader. Il se représente l'art - c'est-à-dire le théatre - selon les mots de Thomas Mann comme «un enchantement, un jeu de couleurs, une fascination intelligente, une danse, une musique et un rêve $»^{2}$. Il succède en 1905 à Otto Brahm à la tête du Deutsches Theater et le dirige jusqu'en 1930. Il ouvre en 1906 à côté du Deutsches Theater une salle de 292 places, les Kammerspiele. L'intérieur est très sobre ; la scène et la salle ne sont séparées que par deux marches. Max Reinhardt veut y donner des œuvres courtes, à peu de personnages, différentes de celles du Deutsches Theater, dans une atmosphère intime et il souhaite rapprocher le plus possible les spectateurs et les acteurs.

3 Max Reinhardt continue à évoluer. On reproche aux Kammerspiele d'être assez chers et d'attirer un public restreint et snob; Max Reinhardt souhaite alors amener au théâtre des gens de tous milieux et retrouver l'esprit du théâtre grec. L'idée d'un théâtre accessible à tous naît à cette époque dans différents pays; elle est défendue en 
particulier par Romain Rolland ${ }^{3}$. Par un paradoxe qui n'est qu'apparent, Max Reinhardt poursuit le même but que dans les Kammerspiele; il souhaite là aussi adapter l'architecture au spectacle et rapprocher, comme nous le verrons, les acteurs et les spectateurs. Il monte pendant l'été 1909 Ædipe roi de Sophocle adapté par Hugo von Hofmannsthal ${ }^{4}$ dans la grande salle du festival de musique de Munich qui contient 3200 places. Il donne dans la même salle L'Orestie d'Eschyle. Il loue ensuite le cirque Schumann à Berlin qui contient 5000 places; il y reprend đEdipe roi le 7 novembre 1910; il y aménage, en la perfectionnant, la même architecture scénique qu'à Munich; la piste correspond à l'orchestra du théâtre grec et des marches mènent à une façade de palais précédée d'un péristyle de six colonnes et d'une plate-forme; un cube encastré dans ces marches rappelle l'autel qui se trouvait dans l'orchestra grec; les spectateurs entourent aux trois quarts les aires de jeu et sont donc près des acteurs; Max Reinhardt fait, comme l'écrit Stern, éclater le cadre de la scène ${ }^{5}$. Le début de la pièce est très spectaculaire: de nombreux figurants qui représentent les Thébains sortent de passages souterrains et supplient Ædipe d'écarter de la ville le fléau. Celui-ci apparaît dans une lumière solaire, symbole de la royauté, et descend vers eux. Après son départ, le chœur arrive à pas mesurés et son allure tranquille contraste avec la brutalité de l'irruption du peuple. Le jeu des acteurs compte moins dans cette immense salle que leur aptitude à s'imposer au public. Paul Wegener a la force nécessaire dans le rôle d'ÆEdipe ; Tilla Durieux, l'interprète de Jocaste, joue un peu trop nerveusement, mais elle est très décorative. Cette représentation remporte un grand succès, mais on reproche parfois à Max Reinhardt d'avoir noyé Sophocle dans sa mise en scène ${ }^{6}$. Max Reinhardt donne ensuite dans le même cirque Schumann en 1911 L'Orestie et Jedermann, miracle du Moyen Âge, dans l'adaptation d'Hofmannsthal. Il prend en 1915 la direction de la Volksbühne qui a le mérite de contenir 2000 places, mais son architecture traditionnelle ne lui convient pas. Il achète alors le cirque Schumann qu'il nomme Grosses Schauspielhaus. Il y fait représenter en 1919 L'Orestie, en 1920 Hamlet, Judith et Holopherne de Nestroy, Danton de Romain Rolland, CEdipe roi, Jules César, Lysistrata et Jedermann, en 1921 Le Marchand de Venise et La Mort de Danton de Büchner. 
Edipe-roi mis en scène par Max Reinhardt au cirque Schumann à Berlin en 1910, Tilla Durieux dans le rôle de Jocaste

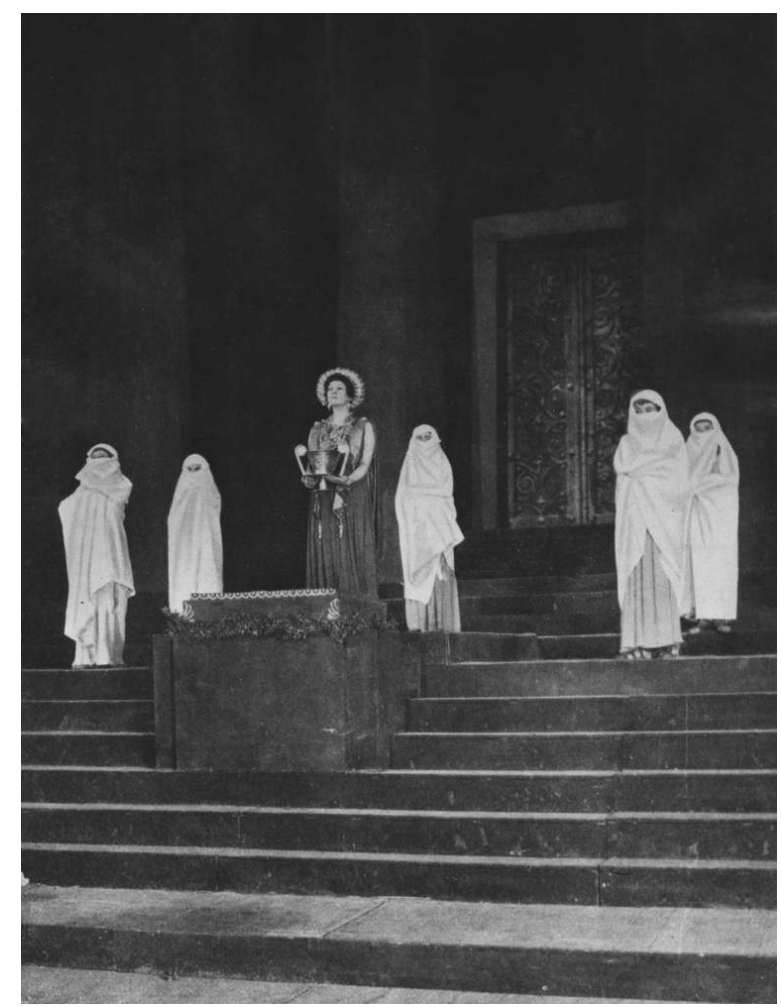

(photo: Archives de l'Akademie der Künste, Berlin).

L'exploitation de la salle s'avère difficile; Max Reinhardt l'abandonne au bout de deux ans et fonde avec Hugo von Hofmannsthal, Richard Strauss et d'autres personnalités le festival de Salzburg. Il veut retrouver l'esprit des représentations du Moyen Âge; il souhaite, comme dans le cirque Schumann, rassembler de nombreux spectateurs et leur révéler de belles œuvres, anciennes et modernes, dans une atmosphère de célébration religieuse et de fête.

5 Max Reinhardt décide de monter Jedermann à Salzburg et forme avec ses amis le projet de transformer la ville en théâtre ${ }^{7}$. Après avoir fait éclater la scène traditionnelle, il s'évade d'un lieu fermé et cherche un cadre de plus en plus large. Jedermann raconte l'histoire d'un mauvais riche, avide de plaisirs ; il finit par se repentir et est sauvé. Max Reinhardt le donne le 22 août 1920 sur la place de la cathédrale pour ouvrir le festival. La cathédrale a une façade en marbre blanc; les bâtiments de la Résidence et du cloître Saint-Pierre bordent la place sur deux côtés. Le regard porte sur les hauteurs voisines, le Mönchsberg et la colline sur laquelle est bâtie la forteresse de Hohensalzburg. Max Reinhardt installe deux podiums juste devant la cathédrale. On accède au premier par sept marches et deux plans inclinés. Un autre podium se trouve derrière, un peu plus haut; il est relié à l'entrée de la cathédrale par une étroite passerelle et muni d'une trappe. Les spectateurs sont assis sur des gradins. Les personnages réels, Jedermann, ses amis et sa maitresse, viennent des arcades qui bordent la place et montent sur le premier podium. Les figures allégoriques sortent de la cathédrale et jouent sur le second ; la Mort sort de la trappe et le Diable vient du public, comme au Moyen Âge. La représentation commence à 17 heures. Les scènes du début qui montrent les appétits terrestres de l'homme se déroulent en pleine lumière. L'ombre des bâtiments 
environnants commence à s'étendre sur la scène lorsque les puissances surnaturelles se manifestent. Le soir tombe au moment de la mort de Jedermann. Des appels «Jedermann, Jedermann» viennent de la forteresse et de la tour de l'église des Franciscains et retentissent sur la ville. Les cloches sonnent quand Jedermann descend dans sa tombe. La ville participe à la représentation et les spectateurs ont l'impression de vivre la pièce.

Max Reinhardt monte Le Grand théâtre du monde de Calderon adapté par Hofmannsthal en 1922 dans la collégiale de Salzburg. La pièce prépirandellienne est une allégorie de la condition humaine. Le dispositif de Max Reinhardt rappelle dans un lieu fermé celui de Jedermann. Une scène à deux niveaux est installée dans le chœur ; des niches sont placées sur le niveau inférieur et des acteurs censés représenter des acteurs qui jouent différents types d'hommes ou de femmes en sortent à l'appel d'un meneur de jeu installé plus haut.

7 Max Reinhardt, tout en dirigeant le festival de Salzburg, prend en 1923 la direction du Theater in der Josefstadt à Vienne. Comme il est d'origine juive, il quitte définitivement l'Allemagne en 1933 quand Hitler prend le pouvoir. Il monte des spectacles dans différentes villes d'Europe et part en 1937 à New-York où il meurt en 1943. Invité en mai $1933 \mathrm{au}$ «Mai musical» de Florence, il monte Le Songe d'une nuit d'été dans les jardins Boboli qui lui fournissent un décor idéal ${ }^{8}$. La représentation a lieu le soir. Les spectateurs sont installés devant une large allée qui s'élève par paliers entre des massifs d'arbres. Un grand bassin, la fontaine de Neptune, se trouve sur le second palier. Reinhardt transforme la nuit réelle en nuit de Shakespeare grâce à une habile utilisation des projecteurs et crée une atmosphère surnaturelle ; les bruits du jardin se mêlent à la musique de Mendelssohn; des kobolds sortent des bosquets et des elfes dansent, Puck et les fées s'envolent. Les costumes Renaissance sont somptueux et le jeu des acteurs italiens très vif.

8 En 1934, Max Reinhardt monte Le Marchand de Venise à Venise même ; la ville réelle et la ville imaginaire ne se distinguent plus. Les spectateurs sont installés sur une place tranquille, le Campo San Trovaso, devant un canal, le Rio Ognisanti; les bâtiments situés sur l'autre rive servent de décor. Le porche majestueux de l'église Saint-Gervais et Saint-Protais est censé être celui du palais de Portia et une maison assez modeste louée par Reinhardt représente celle de Shylock; des gondoles glissent sur le canal. Un pont relie les deux rives; les acteurs le franchissent pour venir devant les spectateurs. L'acteur italien Benassi joue Shylock comme un juif persécuté qui est poussé dans sa tanière et dont la douleur se manifeste d'une manière grotesque.

\section{Gémier}

9 Firmin Gémier, né en 1869, débute dans des mélodrames9. Il joue au Théâtre libre d'Antoine de 1891 à 1894 et au Théâtre Antoine, dirigé par le même Antoine, de 1897 à 1900. Il en prend la direction en 1906 et il la garde jusqu'en 1921.

10 Gémier est lui aussi influencé par Romain Rolland et il désire procurer les joies du théâtre au plus large public possible. Il souhaite, sans employer encore l'expression de théâtre populaire, créer un théâtre qui réunirait des spectateurs des milieux les plus divers. 
11 Il pense que cet élargissement du public implique un nouveau répertoire et de nouveaux lieux théâtraux et qu'il faut revenir, sous une forme ou une autre, au théâtre grec ; il définit ainsi dans Je sais tout (numéro du 15 décembre 1919) la salle dont il rêve :

Ce qu'il faut rétablir, c'est une salle demi-circulaire comme celle de la Grèce antique ou un cirque avec au milieu non pas une piste mais une estrade tel l'échafaud d'autrefois, c'est-à-dire les estrades du Moyen Âge dressées en plein air. Point de décors, de meubles, ni d'accessoires.

12 Gémier loue le Cirque d'Hiver et monte en décembre 1919 Edipe roi de Sophocle dans une adaptation de Saint Georges de Bouhélier. Celui-ci a ajouté des épisodes qui ne figurent pas dans la pièce de Sophocle et les vers sont assez faibles. Gémier essaie de reconstituer dans un lieu couvert un théâtre grec. À l'emplacement de la piste, quelques marches mènent à un espace circulaire qui évoque l'orchestra; un escalier droit coupé par un palier qui part du centre de ce lieu et des escaliers en courbe situés à droite et à gauche conduisent à un péristyle. Ce péristyle est percé de plusieurs portes, une grande au centre et deux autres à droite et à gauche. Il est flanqué de deux ailes. On devine des cours intérieures et des portes et on aperçoit un ciel d'un vert profond qui veut évoquer le ciel grec et des cyprès. Presque toute la pièce se déroule sur les marches de l'escalier. La séparation entre les actes est indiquée par des rideaux gris que des jeunes femmes déploient et emportent en marchant d'un pas rythmé. Gémier recrute pour représenter la foule qui supplie Edipe et les soldats de sa garde centcinquante à deux cents figurants : coiffés de bonnets phrygiens, ils sont divisés en groupes qui portent des costumes d'une même couleur ; le chef de chaque groupe fait des gestes stylisés que les autres imitent.

Gémier intercale entre certains tableaux des intermèdes pendant lesquels les figurants qui jouent les soldats, dont certains sont des athlètes connus, s'adonnent à des exercices athlétiques : lancer du disque et du javelot, lutte. Des danseuses exécutent en même temps, semble-t-il, une danse guerrière, la pyrrhique. Ces intermèdes sont réussis mais ils ralentissent le spectacle et font oublier l'essentiel. Les deux principaux acteurs, Gémier et Andrée Mégard, gênés par les faiblesses du texte et par les dimensions de la salle, ne sont pas tout fait à la hauteur des personnages. Le jeu réaliste de Gémier convient mal à une tragédie. Andrée Mégard est belle et douce mais, comme lui, elle manque de voix.

Gémier monte au Cirque d'hiver le 19 mars 1920 une pièce assez médiocre La Grande Pastorale de Charles Hellem et Pol d'Estoc; le décor fixe est composé d'un mur percé d'une ouverture qui peut représenter divers lieux ; deux escaliers mènent à un balcon.

Gémier ne donne plus d'autres spectacles dans le Cirque d'hiver. Il fonde en novembre 1920 le Théâtre national populaire, ancêtre de celui de Jean Vilar, et il prend un mois plus tard la direction de la Comédie-Montaigne. Malade de la poitrine et épuisé par ses multiples tâches, il meurt en novembre 1933.

\section{Jacques Copeau}

Jacques Copeau (1879-1949) fonde en 1913, sur la rive gauche ${ }^{10}$, le théâtre du VieuxColombier, qui appartient maintenant à la Comédie-Française. Âme de janséniste, il aspire à la pureté et à la rigueur. Il pense que le théâtre de son époque est gâté par le mercantilisme et la routine. Il veut "restituer sa beauté au spectacle scénique", «décabotiniser l'acteur » et jeter un regard neuf sur chaque œuvre ${ }^{11}$. Il supprime tout 
décor et ne garde sur la scène que les objets indispensables. Il ferme le Vieux-Colombier en 1914, le rouvre en 1920 et le ferme définitivement en 1924, pour monter ensuite des spectacles dans des endroits divers. Nous allons examiner quatre d'entre eux, Le Mystère de sainte Olive, Savonarole de Rino Alessi, Comme il vous plaira, joués à Florence en 1933, 1935 et 1938, et Le Miracle du pain doré joué à Beaune en 1943.

Le Mystère de sainte Olive est une œuvre du quatorzième siècle ${ }^{12}$. Le personnage principal, Olive, est une très belle jeune fille que son père, l'empereur de Rome, veut épouser. Elle se brûle les mains dans un brasier pour lui échapper. L'empereur la condamne à mort mais elle parvient à s'échapper, erre dans le monde et est sauvée par la Vierge Marie. Jacques Copeau monte la pièce dans le cloître de la basilique de Santa Croce divisé en quatre carrés par des allées. Son assistant, André Barsacq, aménage une scène octogonale autour d'un puits central. Il place au milieu des carrés quatre platesformes reliées entre elles par des ponts et reliées aussi à la scène centrale; une loggia située au premier étage au-dessus des arcades est censée être l'emplacement du paradis ; les spectateurs sont placés sur des gradins disposés dans la première moitié du pourtour du cloître La mise en scène est très fluide. Les acteurs se déplacent de plateforme en plate-forme au rythme des pérégrinations d'olive ; on apporte parfois sur ces plates-formes quelques éléments de décor que l'on enlève dès qu'olive s'en va. Un chœur d'anges et la Vierge se trouvent dans la loggia. Andrea Pagnani est, d'après le critique Paolo Milano, très simple et très émouvante dans le rôle d'olive. Jacques Copeau sent qu'il est sur la bonne voie et écrit à Suzanne Bing :

Florence n'a pas été seulement un succès. Cela a été une grande expérience et j'ai pu pour la première fois réaliser certaines choses que j'avais trouvées dans mon attente. Maintenant, je me vois mal travailler entre trois murs ${ }^{13}$.

Savonarole de Rino Alessi retrace les principaux épisodes de la vie de Savonarole depuis ses premiers prêches jusqu'à sa mort sur le bûcher ${ }^{14}$. Jacques Copeau le monte sur la place centrale de Florence où Savonarole avait parlé au peuple et où il était mort sur le bûcher. Elle est bordée d'un côté par le Palazzo Vecchio, de l'autre par la loge des Lanzi. Le théâtre rejoue la réalité sans essayer de la reproduire exactement. André Barsacq fait construire au milieu de la place une scène octogonale à deux étages de 80 mètres sur 30 qui rappelle la scène centrale du Mystère de sainte Olive; un orchestre et des chœurs sont installés dans la Loggia dei Lanzi. Toute la ville paraît jouer. On aperçoit un gonfalonier derrière une fenêtre éclairée du Palazzo Vecchio. De nombreux figurants recrutés parmi les habitants de Florence participent au spectacle. Les toits sont chargés de spectateurs.

19 C'est devant la grotte des jardins Boboli que Jacques Copeau monte en 1938, à l'occasion $\mathrm{du}$ «Mai florentin», Comme il vous plaira. Les renseignements manquent sur ce spectacle. Il ne procède pas comme Reinhardt mais utilise des éléments de décor très stylisés qui représentent des arbres ${ }^{15}$.

En 1943 Jacques Copeau monte Le Miracle du pain doré dans la cour des Hospices de Beaune ${ }^{16}$. Le mystère raconte l'histoire d'un mauvais riche, Pierre le changeur, qui est sauvé parce qu'il a donné un jour un pain à un pauvre. La représentation a lieu en fin d'après-midi. L'action principale se déroule sur une scène octogonale qui est censée être la maison de Pierre; elle est entourée d'une plate-forme plus basse qui représente la rue. Deux escaliers arrondis mènent de la scène octogonale à une plate-forme située un peu au-dessus de la galerie qui entoure la cour. Cette plate-forme représente le paradis. Des diables sortent d'un puits situé pour les spectateurs à droite de la scène, un 
orchestre et des chœurs parlants et chantants sont placés en face des spectateurs dans la galerie.

\section{Influences et analogies} son esthétique qu'il juge trop chargée. Pendant la guerre, il voit à New-York un 
spectacle monté par un des collaborateurs de Reinhardt et il note dans son Journal à la date du 5 avril 1917 : «Troisième pièce, Simon the Cyrenia. C'est simple prétexte à une mise en scène à la Reinhardt avec nègres demi-nus et oppositions de couleurs violentes dans les costumes $»^{19}$. En 1933, il trouve que la mise en scène de La Chauve-souris est brillante et superficielle. Le 18 novembre, il n'en adresse pas moins à Reinhardt une allocution aimable dans laquelle il déclare : "Vous avez donné à la vie dramatique plus d'espace et plus d'air, une lumière généreuse, des couleurs brillantes, des proportions plus héroïques et un mouvement plus populaire ${ }^{20}$ ». Même si en 1933 Jacques Copeau était moralement obligé de couvrir Reinhardt de louanges, il nous semble qu'il l'a fort bien compris et qu'il a reconnu son talent. C'est pourquoi, tout en étant moins affirmatif que pour Gémier, nous pensons que l'exemple de Max Reinhardt a stimulé Copeau et l'a aidé à imaginer des espaces théâtraux nouveaux.

\section{Conclusion}

Les innovations de Max Reinhardt se retrouvent à des degrés divers chez Gémier et Copeau qu'il a sans doute inspirés. Ils ont voulu comme lui progresser par un bond de plus de vingt siècles en arrière pour ressusciter l'esprit du théâtre grec. Ils ont éprouvé le même désir de sortir des lieux théâtraux traditionnels pour monter des spectacles soit dans un cirque comme Gémier, soit, comme Copeau, dans des édifices religieux ou en plein air ou dans des décors urbains. Cet élargissement de l'espace convient selon eux à de grands sujets; il embellit la représentation et permet de rassembler de nombreux spectateurs qui se sentent proches des acteurs dans une communauté d'attention et d'admiration. Il serait intéressant de se demander si l'influence de Max Reinhardt s'est exercée sur Jean Vilar par l'intermédiaire de Gémier et de Copeau dont il s'est inspiré. Les courants théâtraux se propagent de pays à pays et ne sont heureusement pas arrêtés par les nationalismes.

\section{NOTES}

1. Nous avons trouvé de nombreux renseignements sur Max Reinhardt dans Leonhard M. Fiedler, Max Reinhardt Reinbeck/Hamburg, Rowohlt, 1975 et Siegfried Melchinger, Max Reinhardt, sein Theater in Bildern, Wien, Friedrich Verlag, 1968. Nous remercions vivement Monsieur Hans Rübesame, archiviste du Deutsches Theater, pour les renseignements qu'il nous a fournis.

2. Nachruf auf Max Reinhardt (article nécrologique sur Max Reinhardt), in Fiedler p. 146.

3. Romain Rolland, Le Théâtre du peuple, Cahiers de la Quinzaine, 1903 ; réédité aux éditions Complexe en 2003.

4. Hofmannsthal s'était servi de deux traductions qui ne figurent ni dans le catalogue de la Staatsbibliothek zu Berlin et ni dans celui de l'Österreichische Nationalbibliothek, l'une de Johann Adam Hartung 1850-1851, l'autre d'Adolf Wilbrandts. Il avait abrégé certains passages et réparti diverses tirades entre plusieurs personnages; cette traduction parut chez S. Fischer à Berlin en 1910 . On peut la consulter dans Hugo Hofmannsthal, Sämtliche Werke,VIII, Frankfurt/Main, S. Fischer,1983. 
5. Ernst Stern, Bühnenbilder bei Max Reinhardt, Berlin, Henschelverlag Kunst und Gesellschaft, 1983, chap. «Sprengung des Bühnenrahmens ». Peter W. Marx, Max Reinhardt, vom bürgerlichen Theater zur metropolitanen Kultur, Tübingen Francke Verlag, 2006. Günther Rühle, Theater in Deutschland 1887-1945, Frankfurt/Main, S. Fisher, 2007, chap. I, p. 162/163.

6. Cf., par exemple, Siegfried Jacobsohn, in Die Schaubühne (17/11/1910) et Norbert Falk, in Fetting, Von der Freien Bühne zum politischen Theater, Band 1, Verlag Philipp Reclam jun. Leipzig 1987 in BZ (Berliner Zeitung) am Mittag, Nr. 262, 8 November 1910.

7. Edda Leisler und Gisela Prossnitz, Hugo von Hofmannsthal, Jedermann. Das Spiel von Sterben des reichen Mannes und Max Reinhardts Inszenierungen, Frankfurt/Main, S. Fischer, 1973.

8. Sur cette représentation et celle du Marchand de Venise, cf. Frederick Tollini, The Shakespeare productions of Max Rreinhardt, Lewiston, NY, E. Mellen Press, 2004 et Walter Zettl, « Max Reinhardt auf der italienischen Szene », in Edda Leisler u.Gisela Prossnitz (éd.), Max Reinhardt in Europa, Salzburg, O.Müller, 1973.

9. Sur Gémier, cf. Paul Blanchart, Firmin Gémier, L'Arche 1954, et Catherine Faivre-Zellner, Firmin Gémier, héraut du théâtre populaire, Presses universitaires de Rennes 2006. Sur ÆEdipe roi, dossier Rt 3738 du département des Arts du spectacle de la BnF (ASP).

10. Sur Copeau, cf. Paul-Louis Mignon, Jacques Copeau, Julliard, 1983.

11. Jacques Copeau, «Un essai de rénovation dramatique : le théâtre du Vieux-colombier », in $N R F, 1^{\mathrm{er}}$ septembre 1913, reproduit in Registres I : Appels, Gallimard, 1974.

12. Fonds Copeau du département des ASP de la BnF, dossier F ${ }^{\circ}-\mathrm{Co}-1 / 774$.

13. Jacques Copeau, Journal, Seghers 1991, tome 2 (1933), p. 352.

14. ASP fonds Copeau F ${ }^{\circ}$-Col-1/605 et dossier Re 5211.

15. ASP fonds Copeau dossier F- COL- $1 / 764$.

16. ASP fonds Copeau, dossiers $\mathrm{F}^{\circ}$-Col- $1 / 424$ et $\mathrm{F}^{\circ}$-Col- $1 / 483$.

17. Cf. Paul Blanchart, op. cit., p. 215.

18. Comœdia 4 sept. 1922 .

19. Jacques Copeau, Journal, Seghers 1991, tome 2, p. 78.

20. Cf. Denis Bablet, « Max Reinhardt in Frankreich », in Edda Leisler u.Gisela Prossnitz (éd.), Max Reinhardt in Europa, op. cit.

\section{RÉSUMÉS}

Dans cette contribution, nous étudierons les similitudes existant dans les mises en scène de trois metteurs en scène qui ont marqué de leur empreinte le théâtre dans la première moitié du $\mathrm{xX}^{\mathrm{e}}$ siècle. Nous mettrons en évidence dans quelle mesure Max Reinhardt a problablement influencé ses collègues français Firmin Gémier et Jacques Copeau. Max Reinhardt avait l'ambition de faire exploser l'espace scénique traditionnel et de rendre le théâtre accessible à un large public. C'est ainsi qu'il réalisa une mise en scène d'Édipe-Roi de Sophocle ainsi que d'autres pièces dans le Cirque Schumann à Berlin qui pouvait contenir jusqu'à 5000 places, Jedermann sur la place de la Cathédrale de Salzburg, Le Songe d'une nuit d'été dans les jardins Boboli de Florence et Le Marchand de Venise à Venise. Firmin Gémier et Jacques Copeau ont suivi cet exemple : Gémier avec une mise en scène de CEdipe-Roi au «Cirque d'Hiver" de Paris, Copeau avec Comme il vous plaira à Florence et avec son Miracle du pain doré dans la cour des hospices de Beaune : la ville, comme ses édifices, servent de décor. L'influence de Reinhardt sur Gémier s'avère incontestable ; en ce qui concerne 
Copeau, elle semble moins évidente, mais sans aucun doute, l'empreinte de Max Reinhardt, dans l'aspiration de ce dernier à créer de nouveaux espaces théâtraux, s'avère déterminante.

Der Beitrag geht den Gemeinsamkeiten in den Bühnenarbeiten von drei Regisseuren nach, die das Regietheater in der ersten Hälfte des zwangisten Jahrhunderts geprägt haben. Es handelt sich dabei vor allem darum, mögliche Einflüsse von Max Reinhardt auf seine französischen Kollegen Firmin Gémier und Jacques Copeau aufzuzeigen. Die Ambition von Max Reinhardt war es, den herkömmlichen Bühnenraum zu sprengen und das Theater einem breiten Publikum zugänglich zu machen. So inszeniert er Sophokles' König EEdipus und andere Stücke im Berliner Zirkus Schumann, der 5.000 Zuschauern Platz bietet, Jedermann auf dem Domplatz von Salzburg, Einen Sommernachtstraum in den Gärten Boboli in Florenz und Den Kaufmann vonVenedig in Venedig. Firmin Gémier und Jacques Copeau folgen diesem Beispiel: Gémier mit einer Inszenierung des König CEdipus im Pariser "Cirque d'hiver», Copeau mit Wie es Euch gefällt in Florenz und mit seinem Miracle du pain doré im Hof des Hospiz von Beaune: die Stadt und ihre Gebaüde dienen jeweils als Dekor. Der Einfluss von Reinhardt auf Gémier erweist sich als unbestreitbar; im Fall von Copeau scheint er wohl weniger evident, aber zweifellos ist Max Reinhardt auch bei dessen Bemühen, neue Theaterraüme zu schaffen, Pate gestanden.

\section{AUTEURS}

\section{PHILIPPE BARON}

Université de Franche-Comté, Besançon 